\title{
ETERNIDAD Y EMANATISMO \\ EN LA GUIA DE PERPLEJOS DE MAIMÓNIDES
}

\section{ETERNITY AND EMANATISM IN MAIMONIDES' \\ GUIDE OF THE PERPLEXED}

\author{
Miquel BeltRÁN* \\ Universidad de las Islas Baleares
}

Resumen: Una de las cuestiones más controvertidas que trata Maimónides en la Guia de Perplejos, que muchos estudiosos han considerado irresoluble, es la de dirimir entre la creación del mundo vs. la eternidad del mismo. La solución a la dicotomía puede encontrarse en la postulación de una creatio ab aeterno que se halla presente en algunos pasajes de la obra, y que se entiende como el sentido esotérico del esquema de la creación que parece describir el Génesis, vinculada — tal postulación — a una peculiar consideración de la voluntad divina, y descrita asimismo como un emanatismo con el que Maimónides identifica la acción de Dios en el universo.

Palabras Clave: Maimónides, eternidad, emanatismo, Dios, voluntad divina, creación.

Авsтract: One of the most puzzling themes of Maimonides' Guide of the Perplexed, that many scholars have considered unresolvable, is the issue of the creation vs. the eternity of the universe. The solution to the dichotomy can be found in a conception of creation ab aeterno, the possibility of which is established in some of the most intricate passages of the book. Maimonides argued in favor of an esoteric sense that stems from a philosophical interpretation of Genesis. This tenet is linked to the conception of God's will held by Maimonides, and also to emanatism, through which the author of the Guide explains God's action on the universe.

Keywords: Maimonides, Eternity, Emanatism, God, Divine Will, Creation.

* La investigación previa a la redacción de este artículo ha sido posible gracias a la participación de su autor, como Investigador Principal, en el Proyecto de Investigación «La influencia de Abraham Cohen de Herrera sobre Spinoza» (FFI2009-07217), financiado por el Ministerio de Ciencia e Innovación. 
Es sabido que la Guía de Perplejos puede considerarse la obra capital en el intento de conciliar la cosmología y física aristotélicas con las creencias judías, pero también la que más claramente muestra hasta qué punto dicho objetivo conduce a una tensión extraordinaria entre doctrinas. No se ignora tampoco que Maimónides distinguió dos niveles de interpretación en su Introducción a la obra, y hasta siete clases o causas de contradicción que podían darse en la forja de un texto, sugiriendo que incumbe al propio filósofo ocultar sus consideraciones esotéricas acerca de la realidad bajo el velo de la doctrina expuesta, con el pretexto del propósito religioso, e incluso del político. La ambigüedad por la que aboga el pensador con respecto a la interpretación de su propia obra alerta a los estudiosos todavía hoy, y éstos debaten entre sí, a veces con poderosos argumentos, sobre cuál sea la concepción verdadera del de Córdoba en torno a asuntos tales como la naturaleza divina, la de la libertad humana, o de cómo tuvo su origen el mundo. También se produce aquella tensión si comparamos la Guía con las otras obras del autor propiamente religiosas o de aleccionamiento del vulgo, como el Mišne Tora. Por cuanto a la cuestión que nos ocupa se refiere, deliberadamente describe Maimónides como oscura e irresoluble la materia de la creación, pero, pese a dicha declarada condición — se trata del Ma’áséh be-rêšrit (Relato de la creación) que junto con el Mảăśèh merkäbāh (Relato del carro celeste) compendia los secretos o doctrinas esotéricas sobre el mundo, esto es, sobre la física y la metafísica-, él mismo se aboca con denuedo a elucidarla.

En Guía II, 13 Maimónides describe tres opiniones sobre la creación. En Guía II, 32 tres más acerca de la profecía, afirmando que lo que piensa la mayoría acerca de esta última es como cuanto concierne a la postulación de la eternidad del mundo, o bien a la relación de la creación con el tiempo y, en efecto, «las opiniones vulgares concernientes a la profecía se asemejan a las relativas a la eternidad o la novación del mundo» ${ }^{1}$. Tal semejanza podría suponer, aventura Rudavsky ${ }^{2}$, que se impone un paralelismo de estricta correspondencia entre las dos cuestiones, en tanto que Maimónides parece equiparar cada una de las opiniones sobre una cuestión con una relativa a la otra. Pero el paralelismo estricto no resulta, a la postre, fácil de argumentar. Si se diese, y éste es el parecer de cierto

1 Guía de Perplejos. Traducción de David González Maeso. Editorial Trotta, 1994. Se cita por el número de la parte, el capítulo correspondiente, y la página. Así, esta primera cita es Guía II, 32, 327.

2 Rudavsky, Tamar M., Time Matters. Time, Creation, and Cosmology in Medieval Jewish Philosophy. State University of New York Press, 2000. Véase en particular el Capítulo Segundo, titulado «Time, Creation and Cosmology», 23-58. 
número de estudiosos ${ }^{3}$, ello daría ocasión de intentar una estrategia que ayude a discernir lo que Maimónides verdaderamente pensaba acerca de la eternidad y de la creación. Nos interesa aquí, ante todo, dar cuenta de la teoría de la eternidad que podemos sostener sobre la exposición, en la obra, del relato de la creación, y ante todo del modo en que Maimónides la enfrenta en la Guía, pero cabe demorarse también en la postulada correspondencia, con el fin de desentrañar su posible condición de fundamento explicativo.

Rudavsky así lo hace y prueba que Maimónides introdujo, en su descripción del secreto de la creación, elementos sustanciales de la eternidad en sentido aristotélico. Esta última doctrina, la de la eternidad en Aristóteles, serviría al propósito de elucidar la sabiduría que el Génesis oculta4. Ya Samuel ibn Tibbon, el primer traductor, en 1204, de la Guía al hebreo, pensó que el secreto de la obra consistía en dar veladamente cuenta de la similitud entre lo que la Ley establece y lo que el estagirita afirmara. Rudavsky, al igual que Klein-Braslavy ${ }^{5}$, reconoce dificultades internas en el sentido literal de la Escritura por lo que se refiere a la temporalidad, pero también que, al no haber argumentos conclusivos en favor de Aristóteles — de quien Maimónides afirmará en Guia II, 17 que no aportó demostraciones de la eternidad — el de Córdoba se habría visto forzado a adoptar un escepticismo epistemológico ${ }^{6}$, que sin embargo equivaldría al tácito rechazo de una fe sostenida en el relato escritutario de la creación.

Al exponer las tres distintas consideraciones sobre el origen del mundo, halla Maimónides que cada una de ellas comporta implicaciones metafísicas ineludibles. Así, la Escritura postula la creación a partir de la absoluta noexistencia (en árabe: min al-'adam). Harvey ya se propuso resaltar la importancia de distinguir esta postulación de la creación ex nibilo. Mientras que la primera en lo usual se interpreta como una continua creación a partir de la no-existencia, lo que Maimónides afirma es que antes de la creación se daba

3 Sobre la controversia es aleccionador el libro de Seeskin, Kenneth, Maimonides on the Origin of the World. Cambridge University Press, 2005.

${ }^{4}$ Cfr. Blumenthal, David R., "Maimonides on Genesis», The Jewish Quarterly Review 72, $1982,223-225$.

5 Klein-Braslavy, Sara, «The Creation of the World and Maimonides' Interpretation of Gen, i-iv», en Schlomo Pines y Yirmiyahu Yovel (eds.), Maimonides and Philosophy, Dordrecht, Martinus Nijhoff Publishers, 1986, 65-78.

6 Según el cual no nos sería dado poder alcanzar la verdad sobre dicha materia.

7 Harvey Warren Z., "A Third Approach to Maimonides' Cosmogony-Prophetology Puzzle», Harvard Theological Review 74, 1981, 287-301. 
la pura no-existencia. La posición escrituraria, de acuerdo con Maimónides, comporta que Dios creó el mundo según su volición le dictaba, que lo hizo a partir de su volición, que lo hizo no a partir de algo (en árabe: la min shay), y que el tiempo es creado. Lo importante de las distinciones anteriores es que la creación que Maimónides vindica es compatible con un universo eternamente carente de principio, esto es, creado ex novo, dependiente de un creador que ha decidido o elegido crearlo, pero no de algo desde lo cual se vería principiado. Harvey piensa que esto es equiparable a argüir que Maimónides sostuvo la posición aristotélica en cuanto a la cosmogonía se refiere, a despecho de su exotérica proclamación de que el mundo fue creado tras la absoluta noexistencia. Pero advierte también Harvey que admitir que Maimónides abogaba por la teoría de la eternidad del estagirita no comporta que negara la creación, ni que se enfrentaría a la Ley; antes rechaza la noción vulgar de creación y la religión tal como la entiende la mayoría. Ya Abulafia ${ }^{8}$ — como este estudioso nos recuerda - observaba en su comentario esotérico a la Guía que es error considerar que la creación y la eternidad del mundo se excluyen mutuamente, pues el mundo puede ser creado y eterno a parte ante (qadmon) ${ }^{9}$. La noción de una creación continua o eterna se hallaría en la misma plegaria de la mañana «Él renueva cada día, continuamente, la obra de la creación». La compatibilidad entre creación y eternidad fue vindicada asiduamente en las obras de quienes comentaban la Guía, en el medioevo. Moisés de Narbona ${ }^{10}$, por ejemplo, el más agudo exégeta de la Guía que jamás haya habido, se refiere a la crucial importancia de la posición por la cual Maimónides vindica la eternidad del mundo como equiparable a la creación eterna (ha-béría ha-nishit).

Con todo, aducir que Maimónides sostenía la doctrina de la eternidad aristotélica no es lo mismo que afirmar que ésta fuese demostrable, pues el de Córdoba deja claro en varios pasajes que considera que ésta no ha sido probada, y que ni siquiera Aristóteles pensó que pudiera serlo. Pero Maimónides se atiene a

8 Sobre Abulafia, cfr. Idel, Moshe, Language, Torah and Hermeneutics in Abraham Abulafia Albany, State University of New York Press, 1989. También Idel, Moshe, «Abulafia’s Secrets of the Guide: A Linguistic Turn», Revue de métaphysique et de morale 4, 8a serie, 1994, 495-528. Asimismo Wolfson, Elliot R., Abraham Abulafia, Kabbalist and Prophet: Hermeneutics, Theosophy, and Theurgy. California, Cherub Press, 2000.

9 Sitre Tora, MS París, heb. 768.

10 Narboni, Moshe, Commentary on the Guide of the Perplexed, Hayoun, Maurice-Reuben (ed.), Tubinga, J. C. B. Mohr, 1986. Sobre Narboni, cf. Hayoun, Maurice-Reuben, La philosophie et la theologie de Moïse de Narbonne (1300-1362), Tubinga, J. C. B. Mohr, 1989. Sobre la cuestión de la eternidad en Maimónides, cfr. Bertola, Hermenegildo, "The Problem of the Eternity of the World in the Thought of Maimonides", Sefarad 56, 1996, 19-43. 
ella —al parecer- por su conformidad con lo que el llama «la naturaleza de la existencia» (en árabe: min tabiat al-wüjud), esto es, con nuestras observaciones empíricas en torno al movimiento continuo de los cielos.

En Guía I, 71 leemos que en las pruebas sobre la existencia, unidad, e incorporeidad de Dios que se detallarán más adelante, en Guia II, 1-2, y en la discusión al respecto en sus obras sobre la ley -Mišne Tora, Yěsode ha-Tora, 1-, el autor presupone la eternidad del mundo con el fin de que la opinión verdadera no se base en un fundamento que cualquiera puede destruir. Leemos en un pasaje que parecería, en primera instancia, auto-contradictorio:

En cuanto a mi método, es como sumariamente voy a describirlo. El mundo, digo yo, necesariamente ha de ser o eterno o creado. Si es creado, indudablemente tiene un Creador, dado que es una noción elemental que lo creado no se ha hecho a sí mismo, sino que otro distinto lo plasmó; por tanto, quien creó el mundo fue Dios. Ahora bien, admitido que el mundo sea eterno, síguese por fuerza, en virtud de tal o tal prueba, que existe otro ser distinto de todos los cuerpos existentes en el cosmos, que no es un cuerpo ni una facultad en un cuerpo, que es uno, permanente y eterno, que no tiene causa y es inmutable: Dios. Ves claramente que las pruebas de la existencia de Dios, de su unidad e incorporeidad han de tomarse necesariamente de la hipótesis de la eternidad del mundo: sólo así será perfecta la demostración, sea eterno el mundo o sea creado. Por eso hallarás que cuantas veces en mis escritos talmúdicos trato de los principios fundamentales e intento establecer la existencia de Dios, recurro a razonamientos orientados hacia la eternidad del mundo; no porque yo crea en ella, sino porque quiero fundamentar $\mathrm{Su}$ existencia... en nuestro credo mediante un método demostrativo en el cual no quepa discrepancia, a fin de no sustentar este dogma verdadero y trascendental sobre una base que cualquiera pueda minar o intentar demoler o tal otro considerarlo como totalmente fantástico ${ }^{11}$.

La hipótesis de la eternidad podría parangonarse, así, a un artificio heurístico. En Guia II, Int., proposición 26 se afirma que el tiempo y la moción son eternos, perpetuos y existentes, y persistentes in actu para los partidarios de la eternidad del mundo, y sin embargo, sabemos que no atañen a Dios. Harvey llega a afirmar que en el Misné Torá la eternidad es condición para el cumplimiento de los mandamientos, al igual que para decir y saber que Dios es uno, y que es sobre

11 Guia I, 71, 195. 
su intuición de la eternidad como Abraham llegó a conocer a Dios. Y en efecto, para varios filósofos medievales, la explicación rabínica de la conversión de Abraham significaba que el patriarca había formulado una prueba filosófica para la existencia de Dios más de un milenio antes de la aparición de la filosofía entre los griegos. Algunos midrāššm ofrecieron durante la Edad Media varias explicaciones, afines entre sí, de cómo llegó Abraham a conocer la existencia divina ${ }^{12}$.

Como apuntábamos, en su elucidación sobre la palabra bara' («creado») que hallamos en el primer versículo del Génesis, Maimónides concluye que ésta no está por la llegada al ser tras la no-existencia (en árabe: ba'd al-'adam), sino por la llegada al ser desde la no-existencia (en árabe: min al-'adam), de lo que se colige que describe tan sólo la continua dependencia óntica de la creación con respecto al creador, o, si se quiere, la información de la materia (por parte de la forma del mundo). La palabra árabe al-bâri” ("Creador») la hallamos en la Guía - ya así lo advierte Harvey - más de veinte veces, en contextos en los que es, al menos, compatible con la teoría aristotélica de la eternidad, pero nunca en un contexto que sugiera que la creación pueda darse tras la no-existencia. El trabajo de rastreo de esta cuestión va más allá del alcance de nuestro propósito aquí, aunque es nuestra intención emprenderlo. En la cuestión de la creación como idéntica a la sola dependencia óntica de lo dado con respecto a Dios nos detendremos más adelante.

Es la creencia de Aristóteles acerca de que el mundo existe en virtud de la necesidad lo que Maimónides rehúsa admitir, puesto que la eternidad concebida de este modo comportaría que la naturaleza no puede verse alterada en su devenir por el designio divino, y que el curso de los eventos permanece inflexible, lo que imposibilitaría la intervención divina sobre el discurrir de los eventos, y ello mina la Ley en su fundamento, reduciendo a la inanidad la esperanza que ésta insufla al permitir la irrupción del milagro. Sin embargo, cuando expone la teoría aristotélica de la creación, Maimónides se refiere a la volición (en árabe: irāda) divina como si fuese compatible con lo que el estagirita sostiene. Tras escribir que

12 Así, por ejemplo, el siguiente: Un día Abraham rezaba al sol. Al atardecer, cuando el sol se puso, Abraham siguió rezándole a la luna. Pero al amanecer también la luna desapareció. Entonces Abraham comprendió que los cuerpos celestes tenían «un Señor sobre ellos», y empezó a adorar al poder invisible que es la verdadera deidad (aunque fuentes hebreas en las que hallamos este relato de la conversión de Abraham son tardías, la historia es muy antigua, y aparece ya en el De Abrahamo, de Filón de Alejandría. Cfr. Filón de Alejandría, Sobre Abraham (De Abrahamo), en Obras Completas. Traducción directa del griego, introducción y notas de José María Treviño, Buenos Aires, 1976, vol. iii, 220-258. 
éste afirmaba que la materia prima, en su esencia, no está sujeta a generación y corrupción, y que a los órdenes superior e inferior «ninguna alteración le(s) acaecerá, que no radique en su naturaleza, ni contingencia alguna, fuera de lo que es consentáneo con su modo de ser, puede en modo alguno acontecerles» ${ }^{13}$, leemos:

Afirma, asimismo, aunque no textualmente, pero se deduce de su opinión, que, a su juicio, es imposible cambie Dios su voluntad o le sobrevenga una nueva, y que a todo este universo, tal como es, Dios le ha dado existencia por su beneplácito, pero no lo sacó de la nada. Piensa, asimismo, que la Divinidad es imposible deje de existir o que su esencia se modifique, como lo es se trueque su voluntad o que le sobrevenga una nueva. En consecuencia, todo este universo, tal como ahora se ostenta, lo fue desde toda la eternidad y así seguirá siendo por siempre ${ }^{14}$.

Pero en Maimónides, también, el poder y la voluntad divinos no existen en el Creador en relación a su esencia ${ }^{15}$, dado que no ejerce poder sobre sí mismo, ni podría atribuírsele una voluntad cuyo objeto sea él mismo, cosa que a nadie —arguye el filósofo- cabría imaginar. Leemos:

Mas bien admiten estos atributos en orden a las diversas relaciones entre Dios y sus criaturas, o sea, que tiene el poder de crear lo que crea, de existenciar lo existente tal como él lo ha realizado, y la ciencia de aquello que realiza. Ves claramente, por tanto, que también estos atributos no deberán referirse a su esencia, sino a las cosas creadas ${ }^{16}$.

La voluntad divina, en Maimónides, es también inmutable:

$\mathrm{Si}$ (la) voluntad pertenece a un ser material y lo apetecido es un objetivo externo, será una voluntad cambiante, a causa de los impedimentos e incidencias, pero la voluntad del ser separado, por nada determinado en modo alguno, no está sujeta a cambio, y si ahora quiere una cosa y mañana otra,

13 Guía II, 13, 270.

14 Guía II, 13, 270.

15 Cfr. a este respecto los recientes y magníficos artículos de Weiss, Roslyn, «Natural Order or Divine Will: Maimonides on Cosmogony and Prophecy», Journal of Jewish Thought and Philosophy 15, 2007, 1-26, y de Manekin, Charles H., "Divine Will in Maimonides' Later Writings", Maimonidean Studies 5, 2008, 189-222.

16 Guía I, 53, 148. 
no implica mutación en su esencia, ni requiere otra causa, como tampoco en que obre o deje de obrar' ${ }^{17}$.

Además, «sólo por equivocidad se aplica por igual a nuestra voluntad y a la del ente separado el término voluntad, no existiendo semejanza entre ambas» ${ }^{18}$. Según Gatti,

el primer elemento teórico que se dirige a vindicar la creatio ab aeterno en Maimónides es precisamente esta concepción de la voluntad divina, que le permite describir la acción de Dios como siguiéndose tan sólo de su sola voluntad, y que el efecto de la sabiduría divina debe ser eterno al igual que lo es la causa, una vez que se admite la identidad, en Él, de su sabiduría y de su esencia ${ }^{19}$.

Ignoramos todo de tal sabiduría y, por tanto, de cómo ella genera el universo. Y prosigue Gatti:

De este modo, Maimónides parece evidenciar el carácter de absoluta auto-referencialidad que la voluntad de Dios posee respecto a la del hombre; es precisamente sobre la base de esta esencial auto-referencialidad como el término "voluntad» resulta equívoco cuando es predicado... de la divinidad (al no poseer los rasgos distintivos de dependencia estructural de lo externo que este término indica cuando viene referido al hombre $)^{20}$.

Con todo, Maimónides no acepta la autoridad de la creencia aristotélica, por no haber sido demostrada, — «ya que no ha sido demostrada no podemos inclinarnos por ella», aduce_- Ciertos estudiosos equiparan aquellas indemostradas consideraciones con la expresión de la doctrina esotérica que Maimónides sostendría, frente a la exposición literal de la escritutaria, que se defiende como doctrina exotérica. Otros sin embargo, como Klein-Braslavy, siguiendo una advertencia de Pines $^{21}$ a tal respecto, arguyen que Maimónides fue en última instancia un escéptico en lo relativo a la posibilidad de concluir algo definitivo acerca de la cuestión,

17 Guía II, 18, 283. Las cursivas son suyas.

18 Guía II, 18, p. 283. Las cursivas son suyas.

19 Gatti, Roberto, «Il problema filosofico della creazione nella Guida dei Perplessi di Maimonide», Materia Giudaica 9, 2004, 175-182, 180.

${ }^{20}$ Gatti, Roberto, 2008, 180.

21 Pines, Schlomo, "Translator's Introduction" to Moses Maimonides' The Guide of the Perplexed, volume one, Chicago, University of Chicago Press, 1963, lvii-cxxxlv. 
y en la Guía, en efecto, Maimónides parece inclinarse por la no-demostrabilidad de cada una de las tres opiniones, alegando que el intelecto humano es incapaz de alcanzar la verdad sobre dicha materia. Ni el propio Aristóteles consideró que su argumento tuviera una fuerza demostrativa definitiva, ni Maimónides lo cree con respecto a su propia defensa de la posición mosaica, tal como la despliegan las páginas de la Escritura. Pero, con respecto a esto último, Rudavsky va más allá y afirma que, si nos detenemos en la consideración sobre el tiempo sostenida por el de Córdoba, habrá que concluir que pensó que el intelecto no puede admitir lo que el Génesis relata. Y que la posición platónica le permitiría conciliar la teoría de la creación con la eternidad, sirviendo de engranaje entre lo que el estagirita pensó y lo que la Ley dicta acerca de la novación del mundo. Así, una lectura sopesada de la posición platónica llevaría a la aceptación de la teoría aristotélica, dando de lado con que ésta parece abogar por la no-necesidad de un creador para el universo, y ello es acorde con la teoría acerca del tiempo que Maimónides sostiene. Se trataría de una versión acomodada de la eterna creación neoplatónica que permite argüir, tal y como Hyman ${ }^{22}$ y Rudavsky han sostenido, la atemporalidad de la creación.

El tiempo, según Maimónides, es un accidente consecuente a la moción, y necesariamente vinculado a ella. La moción sólo existe en el tiempo, y el tiempo no puede ser concebido por el intelecto al margen de la moción. En esto Maimónides sigue la definición aristotélica del tiempo como «medida de la moción». Sostiene que lo que es movido es también creado, viniendo al ser tras no haber sido, algo que parece comportar que Dios creó en primer lugar el tiempo, y después las cosas que se mueven en el tiempo. Nos acercamos a vislumbrar el instante primero de la creación, antes del cual no hubo tiempo. Maimónides está obcecado en argüir que la naturaleza del mundo, tras su haber llegado a la existencia, no se asemeja en nada al estado en que podría imaginarse que se hallara a la espera de ser generado. Y aunque estudiosos como Malino ${ }^{23}$ han observado que las elucubraciones de Maimónides se basan en nociones de temporalidad inaplicables a la determinación del origen del mundo, lo anterior permite al autor de la Guía argüir que la eternidad del mundo Aristóteles la fundamenta

${ }^{22}$ Hyman, Arthur, «Maimonides on Creation and Emanation», en John F. Wippel, (ed.), Studies in Medieval Philosophy, Washington D. C., Catholic University of America Press, 1987, 45-63.

${ }^{23}$ Malino, Jonathan, Maimonides' Guide to the Perplexities of Creation, Tesis doctoral no publicada, presentada en el Hebrew Union College/Jewish Institute of Religion en 1979. También Malino, Jonathan, "Aristotle on Eternity. Does Maimonides Have a Reply?», en Schlomo Pines y Yirmiyahu Yovel (eds.), Maimonides and Philosophy, Dordrecht, Martinus Nijhoff, 1986, 52-64. 
en argumentos, por ejemplo, sobre el imposible paso de Dios de la potencialidad a la actualidad al llevarlo a cabo, que no pueden reconocerse como definitivos, puesto que Dios no actúa en el sentido en que entendemos la acción en términos humanos. En su caso, ocurre que

por la existencia del Creador existe todo, y él es quien perpetúa su duración por una especie de «emanación», como expondremos en uno de los capítulos de este Tratado. Por tanto, si la inexistencia del Creador fuera admisible, tampoco existiría el universo, al desaparecer el elemento constitutivo de sus causas remotas, sus últimos efectos y lo intermediario ${ }^{24}$.

Lo que nos importa de la cita anterior es que no hay acción en Dios como originador del mundo que pueda parangonarse a cómo se actúa en el tiempo. Y que el sentido de emanación postulado en la cita es el único, según Maimónides, que en algo puede aproximarse a explicar que las cosas tengan lugar.

Sus argumentos se basan, de nuevo, sobre la naturaleza equívoca de Dios y la homonimia de los atributos, y propiedades divinas. A diferencia de la volición humana, cuando Dios quiere un cambio no se produce mutación —lo decíamos - en su naturaleza volitiva, lo que comporta que habrá que admitir la perpetuidad de su acción:

Si un agente (otro que Dios)... opera en un tiempo determinado, o deja de operar, puede ser en razón de los impedimentos e incentivos que le sobrevengan o que en él estén. Así, pues, los obstáculos necesariamente le constriñen a abstenerse de hacer lo que desearía, y los incentivos le impulsan a realizar lo que anteriormente no había efectuado. Ahora bien, dado que el Creador.... no tiene incentivos que impliquen un cambio de voluntad, ni obstáculos o impedimentos que puedan sobrevenir o cesar, no existe razón para que opere en un tiempo y en otro no; al contrario, su acción perdura perpetuamente en acto, lo propio que Su permanencia ${ }^{25}$.

Se trata de una volición perpetua que por sí misma hace que la acción divina se dé, de manera incesante ${ }^{26}$, hasta el punto de que los predicados temporales no

24 Guía I, 69, 185.

25 Guía II, 14, 273.

${ }^{26}$ Cfr. A este respecto el magnífico articulo de Agus, Jacob Bernard, "Continuing Creativity in Maimonides' Philosophy», en Abraham I. Katsh y Leon Nemoy (eds.), Essays on the Occasion 
pueden ser usados para describir la actividad de Dios o su naturaleza antes de la creación, pues antes de la misma no existía el tiempo. Por lo tanto, decir que Dios «era» antes de la creación ${ }^{27}$ — siendo «era» indicativo de temporalidad— no tiene sentido y, de modo similar, cualquier pensamiento que se tenga sobre la duración infinita de su existencia antes de la creación del mundo se debe a una suposición errónea, que no se corresponde con su naturaleza. Dios no opera en un contexto temporal, y su misma eternidad es atemporal, de modo que no podrán serle asignados predicados que remitan al tiempo, ni siquiera la duración infinita. Maimónides usa la expresión «imaginar»o «suponer» el tiempo, y sugiere que la doctrina bíblica de la creación comporta un entendimiento vulgar del mismo, basado en la imaginación, que contrasta con la verdadera realidad del tiempo, concordante esta última con la doctrina aristotélica. Pero hemos visto que la teoría del tiempo en Aristóteles induciría a sostener la verosimilitud de la eternidad del universo.

Maimónides afirma — lo decíamos - que Dios trajo a la existencia al mundo sin que ello se diera en un inicio temporal, pues el tiempo es una de las cosas $\operatorname{creadas}^{28}$. Y si admitiéramos la existencia del tiempo antes de la creación, necesariamente nos comprometeríamos con la creencia en la eternidad del mundo. No podemos mantener que la creación es una acción ocurrida según parámetros temporales, porque el mundo no puede haber tenido comienzo, en el sentido de que no tuvo un comienzo en el tiempo. La compleja solución de Maimónides comporta, así, que la acepción de creación a la que se adhiere coexiste con la posibilidad de que el universo, aun siendo innovado, resulte perpetuo (nishit). Se daría una distinción conceptual entre eternidad del universo (qadmut) y su perpetuidad (nishut). La primera es inadmisible en cuanto parece comportar un sustrato del universo co-eterno a Dios. La segunda, sin embargo, es compatible con la idea de que todo deriva de Dios, y no destruye los fundamentos de la Torá. Esta emanación es una suerte de creatio ab aeterno que no introduce contradicción lógica entre el universo creado por Dios, y su perpetua derivación y dependencia óntica de Él, proceso infinito a partir de la Causa Primera.

of the seventeenth Anniversary of the Dropsie University (1909-1979). Filadelfia, The Dropsie University, 1979, 11-32.

${ }^{27} \mathrm{La}$ anterioridad por naturaleza es antagónica, en el Dios maimónideo, a la temporal, y justamente obstaculiza la posibilidad de que el tiempo preceda a la creación.

${ }^{28}$ Filón arguía que el inicio no se asume en sentido temporal sino numérico, de manera que 'en un principio creó' significa que primeramente creó el cielo. Cfr. Filón de Alejandría, Sobre la creación del mundo según Moisés (De Opificio Mundi), en Obras completas. Edición dirigida por José Pablo Martín, Madrid, Editorial Trotta, 2009, vol. I, 95-158. 
En el nivel exotérico, Maimónides prefiere una lectura bíblica literal de la creación. En el esotérico sugiere que una teoría aristotélica del tiempo, por él aceptada, es más consistente con un modelo del universo que lo adscribe a la eternidad, en un sentido compatible con que haya sido, no obstante, creado. Esta lectura se aviene bien con el análisis que Maimónides realiza del término be-rêš̀it. La escritura se inicia con las palabras «en el principio Dios creó» y Maimónides se esfuerza por explicar el sentido de "principio» en este contexto, que él pretende hacer compatible con la creación eterna. La proposición bet en la frase be-rểsìt no es, según su lectura, un indicador temporal, sino que fija el evento en cuestión desde una perspectiva ontológica. Se refiere no a un comienzo temporal, sino a un estado ontológico que subyace. En este sentido, «en el principio Dios creó» describe un evento no-temporal, que sólo especifica que Dios es el creador del universo, esto es, su condición de fundamentador del ser. Según esta interpretación, be-rểsìt refiere un "principio" generador, y se avendría bien con el modo en que Perl ${ }^{29}$ y otros estudiosos han argüido que habría que entender también la dependencia de lo emanado con respecto al Uno en el neoplatonismo, una óptica según la cual el Uno no es algo previo a la diferenciación que establece el ser, sino esta mismo diferenciación que lo constituye y lo distribuye en el desbordamiento de la emanación. Gatti sostiene que en la Guía se dan a la vez dos teorías de la creación, destinadas a dos clases de lectores diferentes: la creación como un acto atemporal en cuyo seno viene instaurado el instante inicial (lo que coincide, tal como el estudioso advierte, con la primera de las tres teorías relativas a la eternidad y la creación del universo que se hallan en Guía II, 13), y la reservada a los intelectos adiestrados en la filosofía, que postula una derivación eterna del universo a partir de Dios. Leemos en Maimónides:

Dios es al universo lo que la forma a lo formalizado, que merced a ella es lo que es, porque la forma es el elemento constitutivo de su verdadera entidad. Tal es la relación de Dios con el mundo, y en este sentido se ha afirmado de él que es «la forma última» y «la forma de las formas»; o sea que él es aquello sobre lo cual descansa, en última instancia, la existencia y estabilidad de todas las formas del mundo y por él subsisten, bien así como las cosas dotadas de forma persisten por ella. Por esta razón se le llamó en nuestra lengua Hay hä-ôläm(îm) («Viviente del mundo»), significando que él es la vida del mundo ${ }^{30}$

29 Perl, Eric D., “"The Power of All Things”. The One as Pure Giving in Plotinus», American Catholic Philosophical Quarterly 31, 1997, 301-313.

30 Guía I, 69, p. 185. 
Dunphy ${ }^{31}$, en el sentido de Rudavsky, ha argüido en varias ocasiones que Maimónides no cree que la creación sea indemostrable ${ }^{32}$, y que muchas de las traducciones modernas de la Guia $a^{33}$ tienden a identificar creación con creación en el tiempo, facilitando así el error en la interpretación de la naturaleza de la misma, error en el que estudiosos tan reputados como Fakhry ${ }^{34} \mathrm{o}$ Wolsfon ${ }^{35}$ habrían caído. Se traduce en ellas de modo que las alternativas se reducen a la eternidad del mundo o bien a su creación temporal. Remite Dunphy a la lectura que hizo Tomás de Aquino de la Guía en la Suma contra los gentiles, 2, 38 y en Suma teológica 1, 46, 2, donde se hace palpable que el aquinate percibió la diferencia sobre la cual puede argüirse que, si bien puede ser demostrado que el mundo se creó ex nibilo, no existen demostraciones válidas acerca de si es eterno o bien tuvo un comienzo temporal. Así, se vindica que una creación ex nihilo eterna no es autocontradictoria. Ello permitiría entender por qué tanto Maimónides —lo hemos visto - como el de Aquino presentan pruebas de la existencia de Dios basadas en la hipótesis de la eternidad del mundo, o que vinculan al «Dios necesario» con la creación del mundo que la Biblia establece.

31 Dunphy, William, "Maimonides and Aquinas on Creation. A Critique of their Historians», en Lloyd P. Gerson (ed.), Graceful Reason. Essays on Ancient and Medieval Philosophy presented to Joseph Owens. Toronto, Pontifical Institue of Mediaeval Studies, 1983, 361-374. También Dunphy, William, «Maimonides not-so Secret Position on Creation», en Eric I. Ormsby (ed.), Moses Maimonides and his Time, Washington D. C., The Catholic University of America Press, 1989, 151-174.

32 Cfr. Gluck, Andrew L., "Maimonides' Arguments for Creation ex nibilo in the Guide of the Perplexed", Medieval Philosophy and Theology 7, 1998, 221-254.

33 Son engañosas, según Dunphy, las traduccions de Friedlander al inglés o la de Munk al francés, por ejemplo, que identifican creación con creación en el tiempo. En cambio, en la magnífica traducción de Pines al inglés el dilema queda expuesto con claridad, de modo que percibimos que la cuestión que se dirime es entre la eternidad del mundo o su creación novedosa. Igual ocurría con la que Giustiniani publicó, cotejando los diversos manuscritos de la traducción al latín, que se hizo, a partir de la segunda traducción al hebreo de la Guía que debemos a al' Harizi, en la década de los 20 del siglo XIII.

${ }^{34}$ Fakhry, Majid, "The "Antinomy" of the Eternity of the World in Averroes, Maimonides, and Aquinas", Le Muséon 66, 1953, 139-155.

35 Wolfson, Harry A., "Hallevi and Maimonides on design, Chance and Necessity", Proceedings of the American Academy for Jewish Research 11, 1941, 105-163, y Wolfson, Harry A., "The Platonic, Aristotelian and Stoic Theories of Creation in Hallevi and Maimonides», en Isidore Epstein, Joseph Herman Hertz, Ephraim Levine and Cecil Roth (eds.), Essays in Honor of the very Rev. Dr. J. R. Hertz, Chief Rabbi of Great Britain, Londres, Edward Goldston, 1942. También Wolfson, Harry A., "The Amphibolous Terms in Aristotle, Arabic Philosophy and Maimonides», Harvard Theological Review 31, 1938, 151-173. Cf. asimismo Wolfson, Harry A., "The Meaning of ex nibilo in the Church Fathers, Arabic and Hebrew Philosophy, and St. Thomas», en Urban T. Holmes y Alex J. Denomy (eds.), Mediaeval Studies in Honor of Jeremiah Denis Matthias Ford Smith, Cambridge, Massachussets, Harvard University Press, 1948, 353-370. 
De este modo, la creación ex nibilo y la creación ex novo son conceptualmente diferentes, pese a que no supieron verlo así eminentes estudiosos como Wolfson. Maimónides itera su preferencia por el método de los filósofos, que inician su disquisición en torno a estas abstrusas materias sobre la naturaleza de la realidad tal como la experienciamos, y a partir de ella establecen qué sea lo que puede demostrarse en torno a Dios como fundamentador de la misma. Tras el examen de dicha realidad, no podemos probar que el mundo sea eterno, de modo que Maimónides se inclina por argüir una posición consistente con su creencia, según la cual la creación es de novo. Y pese a que estudiosos como Davidson ${ }^{36}$ verán contradicciones en su razonamiento, Dunphy demostró hace ya varias décadas que éste no es el caso (Davidson quiso establecer, por ejemplo, que aun diciendo profesar la creencia en un Dios que posee un albedrío libre, Maimónides secretamente sostenía que Dios no ejercita un arbitrio que le permita elegir).

Demorándonos ya en los argumentos concretos de la Guía, Maimónides pensaba que las pruebas más sólidas en favor de la novación del mundo son válidas y se clarifican sólo tras conocer que las inteligencias separadas existen, y tras conocer también el modo en que pueden aducirse pruebas de su existencia ${ }^{37}$. Maimónides advierte a este respecto que no está interesado en las cuestiones filosóficas por ellas mismas, sino con el fin de elucidar los puntos más abstrusos que contiene la Ley, y por hacer manifiesto el significado último de la misma ${ }^{38}$. El universo de los filósofos deriva de la necesidad de la existencia, por medio de una emanación ${ }^{39}$, las diez inteligencias separadas. En Guía II, 6, Maimónides escribe acerca de las disimilitudes y acuerdos entre lo que Aristóteles postula y lo que la Ley establece. En Guía II, 9 afirma el de Córdoba no haber hallado en ningún pensador anterior lo que él descubre por su propia reflexión sobre el

${ }^{36}$ Davidson, Herbert A., "Maimonides' Secret Position on Creation», en Isadore Twersky (ed.), Studies in Medieval Jewish History and Literature. Cambridge, Massachussets, Harvard University Press, 1979, 16-40.

37 «Volveré seguidamente a la prometida argumentación sobre la novedad del mundo, dado que nuestras pruebas más sólidas al respecto solamente serán válidas y claras después de conocida la existencia de dichas inteligencias separadas» (Guía II, 2, p. 247).

38 Cf. Guía II, 2.

39 Pese a que es sin duda a través del neoplatonismo de pensadores como al-Fārābī que Maimónides recibió la doctrina del emanatismo plotiniano, aunque también por las doctrinas ismaelitas con las que entró en contacto tras su llegada a Egipto, el dilema escritutario que está en el origen de su descripción de las diversas consideraciones del origen del mundo, sobre las que discurre, le distancia de estos predecesores. No hallamos que Saadia se demore en esta cuestión, toda vez que sus incursiones en la filosofía las motivaron, en gran parte, doctrinas del pensamiento árabe que le circundaba. 
número cuatro, y procede a desarrollar un posible ordenamiento del universo, tal como sigue:

Nuestro objetivo, en suma, ha sido probar 1ero: que todos los seres existentes, aparte del Creador.... se dividen en tres clases: primera, las Inteligencias separadas; segunda, los cuerpos de las esferas celestes, como un substrato de las formas permanentes, en las que la forma no se transfiere de uno a otro, ni éste es inmutable en su esencia, y tercera, estos cuerpos que nacen y mueren, y van englobados en una misma materia. 2ndo, que el gobierno emana de la Divinidad ... sobre las Inteligencias conforme a su categoría, y que ellas derraman, de lo que han recibido, bienes y luces sobre los cuerpos de las esferas celestes, y éstas difunden energías y beneficios sobre el cuerpo sujeto a generación y corrupción, conforme a la magnitud de los bienes recibidos de sus principios ${ }^{40}$.

La reflexión maimonídea sobre el número cuatro se desarrolla del modo siguiente:

Este número de cuatro es notable y merecedor de reflexión... (así)... todos los Midrāšrim coinciden en afirmar que los ángeles de Dios que vio subir Jacob (por la escala) eran solamente cuatro y no más, «dos que subían y dos que bajan», que los cuatro se juntaban en una de las gradas de la escala, y todos los cuatro se mantenían en una misma línea ${ }^{41}$.

Y más adelante:

En las alegorías de Zacarías, tras la descripción. «Vi cuatro carros que salían de entre dos montes: los dos montes eran de bronce», se añade, a modo de explicación: «Sobre los cuatro vientos del cielo que vienen a presentarse al Señor de toda la tierra».... y que son la causa de todo cuanto se genera $^{42}$.

Habría así cuatro esferas, los elementos emanados de ellas — asimismo cuatro- y las fuerzas que emanan de éstos hacia lo que existe en el mundo sublunar, que son cuatro también. Del mismo modo este número juega un importante

40 Guía II, 11, p. 263.

41 Guia II, 10, p. 261.

42 Guia II, 10, p. 261. 
papel en la explicación de la actividad causal de las esferas, y vincula la acción de éstas con pasajes de la Escritura y del Talmud que refieren cuatro órdenes de ángeles, o aluden a los cuatro ángeles vistos por Jacob, o también a los cuatro carros interpretados por Zacarías como cuatro «vientos» que los cielos poseen, y que son, como leíamos, la causa de todo lo que se da en el tiempo ${ }^{43}$.

Estas numeraciones estarían por el hecho de que bajo el Creador existen, en un decreciente orden de perfección e importancia, el reino de las inteligencias separadas, el de las esferas celestiales, y el mundo sublunar de las entidades generadas y corruptibles, pero se vinculan con que en Guía II, 11 se introduzca la vindicación de una concepción especial de la emanación:

La emanación procedente de Dios... para producir las inteligencias separadas, se comunica también de éstas para que unas produzcan a otras, hasta el intelecto activo, en el que finaliza la producción de las mismas. De cada una fluye asimismo otra producción hasta que las esferas terminan en la luna, tras la cual adviene este cuerpo sujeto a generación y corrupción, a saber, la materia prima, y lo que de ella está compuesto. De cada esfera penetran fuerzas en los elementos hasta que su efusión se detiene en el proceso de generación y corrupción ${ }^{44}$.

Más importante es que en Guía II, 12 se declara:

No hay metáfora más apropiada que esta expresión, «emanación», para significar la acción del ser separado.... (puesto que).... Dios es incorpóreo, y ni aun se acerca a lo que hace, imaginaron que Él intima órdenes a los ángeles, y éstos cumplimentan los actos mediante contacto y aproximación de un cuerpo con otro, tal como hacemos nosotros, y de ahí dedujeron que los ángeles son también cuerpos. ${ }^{45}$

Tanto éstos como los que creen que Dios manda una cosa hablando, están sumidos en un "puro proceso imaginativo» del que derivan todos los vicios racionales o morales, según Maimónides.

43 Guía II, 10, p. 261.

44 Guía II, 11, p. 264.

45 Guia II, 12, pp. 266-267. 
Si Guía II, 11 se abría arguyendo que la emanación se describe como el modo de causalidad propio de ciertas causas eficientes, en concreto, el Creador y las inteligencias separadas o ángeles, en el capítulo 12 se lee que el término se ha aplicado a Dios en el lenguaje hebreo por usar el símil de una fuente o manantial de agua. La razón de este uso es la extrema dificultad, si no imposibilidad, de la que adolece el intelecto humano, moldeado como está por la facultad imaginativa -que sólo puede representarse seres corpóreos y las acciones que se dan entre ellos- de concebir cómo un ser inmaterial actúa. El término fue usado en este sentido figurado por los profetas, precisamente por la aproximación con que éste permite vislumbrar el discurrir de la acción divina, por ejemplo en Jeremías 2, 12: «Pasmaos, cielos, de esto.... y horrorizaos.... sobremanera, oráculo de Yavé, pues un doble mal ha cometido mi pueblo: dejarme a mí, la fuente de aguas vivas, para excavarse cisternas agrietadas, incapaces de retener el agua», o Salmos 36, 10: «Porque en ti está la fuente de la vida, en tu luz vemos la luz». Maimónides, a su vez, escribe:

Su acción es constante, y siempre que se da un factor determinativo, recibe esa acción perpetua designada con el nombre de emanación... con respecto al Creador,... demostrada Su incorporeidad, que el mundo es obra suya, y que Él es su causa eficiente, conforme expusimos y después insistiremos, se ha afirmado que el mundo proviene de una emanación del Creador, que Él ha irradiado, sobre el mismo todo cuanto en Él adviene ${ }^{46}$.

El sentido es el mismo que cuando se escribe que derrama su ciencia sobre los profetas, respecto a cuyos textos, la creencia en la incorporeidad de Dios —aduce Maimónides - no desmiente ninguna de sus partes. También muchos pasajes del Pentateuco y otros libros se entienden en conformidad con la teoría platónica, que admite la eternidad, pero que no la envuelve de necesidad aristotélica y, por tanto, permite estimar que la creación del mundo fue ex nibilo, tras su absoluta inexistencia:

Según exigencia de nuestra religión, que atribuye la existencia y desaparición de las cosas a la voluntad de Dios... no a la necesidad,... nada nos obliga a admitir que Dios.... después de haber existenciado una cosa antes inexistente, debe obligatoriamente destruirla; más bien dependerá de su voluntad

46 Guia II, 12, p. 266. 
libre de destruirla o conservarla, o bien de lo que exija su sabiduría ${ }^{47}$. De consiguiente, es posible que lo conserve perpetuamente y le otorgue una permanencia semejante a la de $\mathrm{El}^{48}$

También en la doctrina expuesta por Salomón en los libros sapienciales a él atribuidos aparecen algunos pasajes que dan a entender la perpetuidad del mundo

y a la vista de tales afirmaciones se pensó creía Salomón que el mundo era increado. Pero no es así.... Él mismo aseveró... que esas obras de Dios, a saber, el mundo, y lo que él encierra, perdurarán para siempre en su naturaleza, a pesar de haber sido creadas: "Cuanto hace Dios es permanente, y nada se le puede añadir, ni quitar» $(E c l 3,14)$. Muestra, pues, en este versículo, que el mundo es obra de Dios y perdurable, y adujo asimismo la razón de su perpetuidad al decir: «nada se le puede añadir ni quitar», porque tal es la causa de su perdurabilidad. Como si dijera que lo sujeto a mutación cambia por razón de algún defecto ínsito en ello, y debe corregirse, o bien de aquello que tuviere de superfluo e inútil, y deba retraerse. Ahora bien, las obras de Dios, .... siendo perfectas, sin posibilidad de añadirles ni sustraerles nada, permanecen necesariamente tal como son, inmunes a todo cambio...Al decir después, "Lo que es, eso fue ya, y lo que fue, eso será, y Dios busca esta continui$d_{a d »}(E c l 3,15)$, quiere significar que... desea la perpetuidad del cosmos, y que sus componentes conserven su mutua concatenación. Lo dicho sobre la perfección de las obras de Dios..., como de la imposibilidad de añadirles ni quitarles nada, el Príncipe de los Sabios lo declaró en estos términos, «Él es la Roca: sus obras son perfectas» Dt 32, 4), es decir, que toda su obra, a saber, sus criaturas, son absolutamente perfectas, sin mezcla alguna de defecto, y sin nada superfluo o inútil ${ }^{49}$.

Maimónides pensó que el poder de la voluntad divina es suficiente para traer las cosas a la existencia, lo que comporta que la génesis de todas ellas es a partir de Dios, y que todas dependen de él en este sentido, algo que podría suponer que la creación del mundo estaba en la mente divina desde la eternidad, pero que antes de que lo creara se daba la absoluta no-existencia, lo que significaría que Dios

47 Ambas cosas son una si admitimos que su voluntad y su sabiduría son idénticas con su esencia.

48 Guia II, 27, pp. 306-307.

49 Guía II, 28, pp. 308-309. 
lo creó por un proceso emanativo como el concebido por Plotino, excepto que la emanación en Maimónides tuvo un comienzo y fue, a diferencia de lo que el autor de las Enéadas pensara, fruto del designio de la voluntad divina.

Que el proceso emanativo por el que lo múltiple deriva de lo uno no puede explicarse es una constante que recurre en las páginas de la Guía, en la que se itera también que no podemos llegar a alcanzar cuál sea el contenido de la sabiduría divina. La creación como producto de la volición la interpreta Maimónides como una procesión a partir de Dios, y ex nibilo significaría justamente eso, «a partir de Dios». Pero la pura inexistencia que es previa a la creación del mundo sería entonces identificable con Él, lo que comportaría la incomprehensibilidad de su esencia o la imposibilidad de que haya un concepto en nuestro intelecto que lo refiera $^{50}$, y lo postula asimismo como la más notoria prefiguración del término Ayin ${ }^{51}$ con que en la cábala se nombra a la Causa Primera.

\section{Bibliografía}

Agus, Jacob Bernard (1979). "Continuing Creativity in Maimonides' Philosophy», en Abraham I. Katsh y Leon Nemoy (eds.), Essays on the Occasion of the seventeenth Annivesary of the Dropsie University (1909-1979). Filadelfia: The Dropsie University, 11-32.

Bertola, Hermenegildo (1996). "The Problem of the Eternity of the World in the Thought of Maimonides», Sefarad 56, 19-43.

Blumenthal, David R., (1982). «Maimonides on Genesis», The Jewish Quarterly Review 72, 223-225.

Burrel, David B. (1986). Knowing the Unknowable God. Ibn Sina, Maimonides, Aquinas. Notre Dame, University of Notre Dame Press.

Davidson, Herbert A. (1979). «Maimonides' Secret Position on Creation», en Isidore Twersky (ed.), Studies in Medieval Jewish History and Literature. Cambridge, Massachusetts, 16-40.

- (1987). Proofs for Eternity, Creation, and the Existence of God in Medieval Islamic and Jewish Philosophy, Nueva York, Oxford University Press.

50 Cf. Burrell, David B., Knowing the Unkowable God. Ibn Sina, Maimonides, Aquinas. Notre Dame, University of Notre Dame Press, 1986.

${ }^{51}$ Cfr. Sobre esta consideración Daniel C. Matt, «Ayin: The Concept of Nothingness in Jewish Mysticism», en Lawrence Fine (ed.), Essential Papers on Kabbalah, New York University Press, 2000, 67-108. 
Diamond, James Arthur (2002). Maimonides and the Hermeneutics of Concealment. Deciphering Scriptures and Midrash in the Guide of the Perplexed. Albany, New York, SUNY Press.

Dunphy, William (1983). «Maimonides and Aquinas on Creation. A Critique of their Historians», en Lloyd P. Gerson (ed.), Graceful Reason. Essays on Ancient and Medieval Philosophy presented to Joseph Owens. CSSR, Toronto, 361-374.

- (1989). «Maimonides not-so Secret Position on Creation», en Eric I. Ormsby (ed.), Moses Maimonides and his Time, Washington, The Catholic University of America Press, 151-174.

Filón de Alejandría (1976) Obras completas. Traducción directa del griego, introducción y notas de José María Treviño, vol. iii, Buenos Aires.

- (2009). Obras completas, edición dirigida por José Pablo Martín, Madrid, Editorial Trotta, vol. i.

Fackenheim, Emil L. (1946-47). «The Possibility of the Universe in Al-Farabi, Ibn Sina and Maimonides», Proceedings of the American Academy for Jewish Research 16, $39-70$.

FAKHRY, Majid (1953). «The 'Antinomy' of the Eternity of the World in Averroes, Maimonides and Aquinas», Le Muséon 66, 139-155.

Feldman, Seymour (1990). «Abravanel on Maimonides' Critique of the Kalam Arguments for Creation», Maimonidean Studies 1, 15-25.

GaTTI, Roberto (2004). «Il problema filosofico della creazione nella Guida dei perplessi di Maimonide», Materia Giudaica 9, 175-182.

Gluck, Andrew L. (1998). "Maimonides' Arguments for Creation ex nibilo in the Guide of the Perplexed", Medieval Philosophy and Theology 7, 221-254.

Goldstein, Jonathan A. (1984). «The Origins of the Doctrine of Creation ex nibilo», Journal of Jewish Studies 35, 127-135.

Harvey, Warren Z. (1981). «A Third Approach to Maimonides' Cosmogony-Prophetology Puzzle», Harvard Theological Review 74, 287-301.

Hyman, Arthur (1988). «Maimonides on Creation and Emanation», en John F. Whippel (ed.), Studies in Medieval Philosophy, Washington D.C., Catholic University of America Press, 45-61.

Idel, Moshe (1989). Language, Torah and Hermeneutics in Abraham Abulafia. Albany, State University of New York Press.

- (1994). «Abulafia’s Secrets of the Guide: A Linguistic Turn», Revue de métaphysique et de morale 4, $8^{a}$ serie, 495-528. 
Klein-Braslavy, Sara (1986). "The Creation of the World and Maimonides' Interpretation of Gen i-iv», en Schlomo Pines y Yirmiyahu Yovel (eds.), Maimonides and Philosophy, Dordrecht, Martinus Nijhoff Publishers, 65-71.

Kogan, Barry S. (1988). «The Problem of Creation in Late Medieval Jewish Philosophy», en Ruth Link-Salinger (ed.), A Straigh Path: Studies in Medieval Philosophy and Culture, Washington D. C., The Catholic University of America Press, 159-173.

Mosé Ben Maimón (Maimónides 1994), Guía de Perplejos, traducción de David González Maeso. Editorial Trotta.

Malino, Jonathan W. (1979). Maimonides' Guide to the Perplexities of Creation. Tesis doctoral no publicada, HUC.

- (1986). "Aristotle on Eternity: does Maimonides have a Reply?», en Schlomo Pines and Yirmiyahu Yovel (eds.), Maimonides and Philosophy, Dordrecht, Martinus Nijhoff Publishers, 52-64.

Manekin, Charles H. (2008). «Divine Will in Maimonides' Later Writings», Maimonidean Studies 5, 189-222.

Matт, Daniel C. (2000) «Ayin: The Concept of Nothingness in Jewish Mysticism», en Lawrence Fine (ed.), Essential Papers on Kabbalah, New York University Press, 67-108.

Hayoun, Maurice-Reuben (1994). Mä̈monide et la pensée juive. París, Presses Universitaires de France.

- (1989). La philosophie et la théologie de Mö̈se de Narbonne (1300-1362), Tubinga, J. C. B. Mohr.

Narboni, Moshe (1986). Commentary on the Guide of the Perplexed. Maurice-Reuben Hayoun (ed.), Tubinga, J. C. B. Mohr.

Perl, Eric D. (1997). "The Power of All Things': The One as Pure Giving in Plotinus», American Catholic Philosophical Quarterly 31, 301-313.

Pines, Schlomo (1963). "Translator's Introduction» to Moses Maimonides' The Guide of the Perplexed, volume one, Chicago, University of Chicago Press, lvii-cxxxlv.

Rudavsky, Tamar M. (1997). "Creation and Temporality in Medieval Jewish Philosophy», Faith and Philosophy 14, 458-477.

- (2000). Time Matters. Time, Creation, and Cosmology in Medieval Jewish Philosophy. Nueva York, State University of New York Press.

Samuelson, Norbert M. (1991). "Maimonides' Doctrine of Creation», Harvard Theological Review 84, , 249-271.

SeEsKin, Kenneth (2003). Maimonides on the Origin of the World. Cambridge University Press. 
Tomás de Aquino (1998). Suma contra los gentiles, 4. ${ }^{a}$ edición, México, Editorial Porrúa, S.A.

- (1989). Suma de Teología, 2a edición, Madrid, BAC.

Weiss, Roslyn (2007). «Natural Order or Divine Will: Maimonides on Cosmogony and Prophecy», Journal of Jewish Thought and Philosophy 15, 1-26.

Wolfson, Harry A. (1938). "The Amphibolous Terms in Aristotle, Arabic Philosophy and Maimonides», Harvard Theological Review 31, 151-173.

- (1941). "Hallevi and Maimonides on Design, Chance and Necessity», Proceedings of the American Academy for Jewish Research 11, 105-163.

- (1942). «The Platonic, Aristotelian and Stoic Theories of Creation in Hallevi and Maimonides», en Isidore Epstein, Joseph Herman Hertz, Ephraim Levine, Cecil Roth (eds.), Essays in Honor of the very Rev. Dr. Hertz, Chief Rabbi of Great Britain, London, Londres, Edward Goldston.

- (1938). "The Amphibolous Terms in Aristotle, Arabic Philosophy and Maimonides», Harvard Theological Review 31, 151-173.

- (1948). "The Meaning of ex nibilo in the Church Fathers, Arabic and Hebrew Philosophy, and St. Thomas», en Urban T. Holmes y Alex J. Denomy (eds.), Mediaeval Studies in Honor of Jeremiah Denis Matthias Ford, Cambridge, Massachusetts, Harvard University Press, 353-370.

- (1970). "The Identification of ex nihilo with Emanation in Gregory of Nyssa», Harvard Theological Review 63, 53-60.

Wolfson, Elliot R. (2000). Abraham Abulafia, Kabbalist and Prophet: Hermeneuticcs, Theosophy, and Theurgy. California, Cherub Press.

Recibido: 10/12/2012

Aceptado: 7/02/2013 